\title{
Sobrevivência em viveiro de mudas de espécies nativas retiradas da regeneração natural de remanescente florestal
}

\author{
Ricardo Augusto Gorne Viani( ${ }^{(1)}$ e Ricardo Ribeiro Rodrigues ${ }^{(2)}$
}

\begin{abstract}
(1)Universidade Estadual de Campinas, Instituto de Biologia, Dep. de Botânica, Caixa Postal 6109, CEP $13083-970$ Campinas, SP. E-mail: viani@unicamp.br (2)Escola Superior de Agricultura Luiz de Queiroz, Dep. de Ciências Biológicas, Av. Pádua Dias, 11, Caixa Postal 9, CEP 13418-900 Piracicaba, SP. E-mail: rrr@esalq.usp.br
\end{abstract}

\begin{abstract}
Resumo - O objetivo deste trabalho foi avaliar a sobrevivência em viveiro de plântulas retiradas da regeneração natural de remanescente de Floresta Estacional Semidecidual, localizado em Bofete, SP. Indivíduos com até $30 \mathrm{~cm}$ de espécies arbustivo-arbóreos foram coletados em parcelas instaladas previamente em remanescente florestal, transplantados para viveiro sob sombrite $50 \%$, e avaliados periodicamente durante nove meses. Ao todo foram transferidos para o viveiro 2.424 indivíduos, pertencentes a 110 espécies. A taxa média de sobrevivência foi de $69 \%$, com variação nos resultados para as diferentes espécies, famílias e classes de altura dos indivíduos. Embora espécies pioneiras tenham apresentado taxa de sobrevivência significativamente maior que não pioneiras, várias espécies não pioneiras apresentaram elevada taxa de sobrevivência. Muitas das espécies sobreviventes não são encontradas nos viveiros florestais do Estado de São Paulo. A produção de mudas por meio da transferência da regeneração natural de áreas naturais é uma estratégia complementar viável, que eleva a riqueza de espécies dos viveiros florestais.
\end{abstract}

Termos para indexação: produção de mudas, plântulas, transplante de plântulas, restauração florestal.

\section{Survival in nursery of native species saplings obtained from natural regeneration of forest fragments}

\begin{abstract}
Seedling survival was evaluated regarding transplanted saplings obtained from natural regeneration of a Semideciduous Seasonal Forest fragment, located at Bofete, São Paulo State, Brazil. Shrub and trees species seedlings up to $30 \mathrm{~cm}$ height were collected from samples sited on a forest remnant, transplanted to a nursery under 50\% shade tissue, and evaluated periodically during nine months. A total of 2,424 seedlings, belonging to 110 species were transferred to the nursery. Average survival reached $69 \%$, although this survival rate was quite variable according to the species, families and height classes of the evaluated individuals. In spite of the fact that pioneer species presented higher survival rate, several non-pioneer species also obtained high values of survival. Besides, many of the survivor species are usually not available in forest nurseries of the São Paulo State. The transference of seedlings from natural regeneration of forest fragments to forest nurseries seems to be a complementary and a viable method in order to produce saplings of native species, increasing diversity within nurseries with forest restoration purposes.
\end{abstract}

Index terms: sapling production, seedlings, seedlings transplanting, forest restoration.

\section{Introdução}

A produção de mudas florestais com qualidade, quantidade e diversidade suficiente é uma das fases mais importantes para o estabelecimento de bons povoamentos com espécies florestais nativas (Gonçalves et al., 2000). Entretanto, a obtenção de mudas de diversas espécies do ambiente regional em quantidade suficiente para o plantio é o primeiro e um dos principais pontos de estrangulamento dos programas de restauração ecológica de determinada área (Fonseca et al., 2001; Santarelli, 2004).
Há um deficit de sementes e conseqüentemente de produção de mudas de espécies nativas com alta diversidade para uso na recuperação de áreas degradadas. A análise recente de 30 viveiros florestais do Estado de São Paulo revelou que cerca de 340 espécies arbóreas nativas são produzidas. A maioria deles, no entanto, concentra sua produção em apenas 30 espécies, as quais são as prontamente disponíveis e com tecnologia de produção conhecida e por isso as que predominam nos reflorestamentos (Barbosa et al., 2003). Isto ocorre em razão das dificuldades de obtenção de sementes nativas 
e da ausência de tecnologia específica para produção de mudas de muitas das espécies nativas (Silva et al., 2003; Zamith \& Scarano, 2004).

A variabilidade interespecífica, a presença de diversificados mecanismos de dormência (VázquesYanes \& Orozco-Segovia, 1993; Oliveira et al., 2003), os altos índices de predação (Zamith \& Scarano, 2004) e o conhecimento limitado sobre a fenologia e a fisiologia de parte significativa das plantas arbustivo-arbóreas tropicais são barreiras que dificultam a produção de mudas de espécies nativas.

Por isso, métodos que incrementam a diversidade e a disponibilidade de mudas para a restauração de áreas degradadas, como a produção de mudas a partir da coleta e transferência dos indivíduos da regeneração natural em formações florestais nativas, passaram a ser recomendados (Rodrigues \& Gandolfi, 2004).

Embora apresente vantagens como a eliminação de etapas trabalhosas do processamento de sementes e a possibilidade de se produzir mudas de espécies com inconvenientes de dormência de sementes ou mesmo de espécies com baixa produção e/ou dificuldade de coleta de sementes, a produção de mudas usando a transferência de indivíduos de regeneração natural é pouco expressiva no Brasil (Corvello, 1983).

Estudos sobre a utilização de propágulos alóctones originados da regeneração natural na produção de mudas e na restauração ecológica, de maneira geral, apresentam resultados de sobrevivência dos indivíduos satisfatórios e recomendam essa técnica como viável para a produção de mudas de espécies nativas (Auer \& Graça, 1995; Nemer et al., 2002). Entretanto, os estudos são escassos e restritos a poucas espécies nativas.

O objetivo deste trabalho foi avaliar a sobrevivência da comunidade de plântulas arbustivo-arbóreas, transplantadas da regeneração natural de remanescente florestal para viveiro de produção de mudas florestais e o potencial deste procedimento como técnica de produção de mudas de espécie nativas.

\section{Material e Métodos}

As mudas foram obtidas em remanescente de vegetação secundária de Floresta Estacional Semidecídua $\left(23^{\circ} 0^{\prime}-23^{\circ} 5^{\prime} \mathrm{S}\right.$ e $\left.48^{\circ} 11^{\prime}-48^{\circ} 16^{\prime} \mathrm{W}\right)$, com aproximadamente 150 ha, localizado na Fazenda Santa Terezinha, Bofete, SP, numa região de clima Cfa segundo classificação de Köppen, precipitação pluvial em torno de $1.400 \mathrm{~mm}$ anuais e cerca de $600 \mathrm{~m}$ de altitude. $\mathrm{Na}$ área de coleta, o remanescente florestal abrange desde áreas de interflúvio, sem nenhuma influência fluvial até locais de vegetação ribeirinha com influência sazonal quase permanente.

$\mathrm{Na}$ coleta das plântulas, foram instaladas aleatoriamente, no remanescente florestal, 80 parcelas de $2 \times 2 \mathrm{~m}$. Nessas parcelas, durante abril e maio de 2003, todos os indivíduos arbustivo-arbóreos com até $30 \mathrm{~cm}$ de altura foram transferidos para viveiro.

As plântulas foram extraídas do solo com auxílio de pá de jardinagem, com posterior destorroamento manual até ficar com as raízes nuas. Imediatamente após, as plântulas foram colocadas em recipientes com água, sendo mantidas nesta condição até o transplante, realizado em viveiro localizado na Fazenda Santa Terezinha, próximo ao local de coleta.

Foram transplantadas para o viveiro 2.424 plântulas pertencentes a 110 espécies arbustivo-arbóreas. No processo de transplante, os indivíduos foram acondicionados em sacos pretos de polietileno $(10 \mathrm{x} 15 \mathrm{~cm})$ e mantidos sob sombrite $50 \%$. Foi utilizado substrato à base de vermiculita expandida e de cascas vegetais e turfas processadas e enriquecidas. Raízes excessivamente grandes ou tortas foram podadas quando maiores que o recipiente de transplante. No momento do transplante foi realizado também o corte de $50 \%$ da superfície de cada folha dos indivíduos regenerantes. A disposição das plântulas no viveiro foi aleatória.

Os tratos culturais realizados foram os mesmos comumente utilizados na produção de mudas florestais, ou seja, irrigações por aspersão duas vezes ao dia e controle manual de ervas daninhas. A partir dos 30 dias até o término das avaliações, foram realizadas adubações líquidas mensais, com $92 \mathrm{~g}$ de carbonato de cálcio, $50 \mathrm{~g}$ de fosfato monoamônico (MAP), $35 \mathrm{~g}$ de cloreto de potássio, $26 \mathrm{~g}$ de uréia, $44 \mathrm{~g}$ de sulfato de magnésio e $22 \mathrm{~mL}$ de solução de micronutrientes, diluídos em $40 \mathrm{~L}$ de água.

Uma vez transplantadas, as espécies foram identificadas segundo APG II (Angiosperm Phylogeny Group, 2003) e classificadas sucessionalmente em pioneiras, incluindo pioneiras e secundárias iniciais, e não pioneiras, incluindo secundárias tardias e clímax, de acordo com classificações existentes em Ferretti et al. (1995) e Ivanauskas et al. (2002). Espécies não enquadradas nas categorias acima ou sem dados disponíveis foram denominadas não caracterizadas. 
Logo após o transplante, procedeu-se a medição da altura dos indivíduos, do colo à inserção da última gema. Quanto aos indivíduos da família Arecaceae, a altura foi tomada medindo-se do nível do solo até a extremidade da folha mais alta, com a plântula posicionada na vertical.

Este mesmo procedimento foi repetido aos três, cinco, sete e nove meses após o transplante. Nessas avaliações, os indivíduos foram também classificados em sobreviventes e mortos. Indivíduos que até a última avaliação não haviam emitido ou estavam desprovidos de folhas foram considerados mortos.

Foram calculadas taxas de sobrevivência por espécie, por família, por estágio sucessional e geral. A taxa de sobrevivência geral foi calculada com e sem a espécie Protium spruceanum, em razão de esta corresponder a mais de 50\% das plântulas da área de coleta, o que influencia os resultados e dificulta sua interpretação. As proporções de sobreviventes e mortos por estágio sucessional (não pioneiras e pioneiras) foram comparadas pelo teste qui-quadrado, com correção de Yates $(\mathrm{GL}=1)$ (Beiguelman, 1994).

Os indivíduos foram ainda separados em classes de altura, instituídas de forma arbitrária: classe I, quando até $10 \mathrm{~cm}$, classe II, quando de 11-20 cm e classe III, quando de 21-30 cm. A proporção de sobreviventes e mortos em cada classe de altura foi comparada nos dados gerais, nos diferentes estágios sucessionais e para Protium spruceanum isoladamente. Para tanto, utilizou-se o teste qui-quadrado, comparando as classes duas a duas, com a correção de Yates $(\mathrm{GL}=1)$ e a correção de Bonferroni para comparações múltiplas. Nos casos de freqüências inferiores a cinco, o teste qui-quadrado foi substituído pelo teste exato de fisher (Beiguelman, 1994).
Em cada época de avaliação, foram calculadas taxas de mortalidade e porcentagem dos indivíduos sobreviventes que haviam emitido folhas e, por fim, a lista de espécies sobreviventes após o período de avaliação foi comparada à de espécies levantadas em viveiros do Estado de São Paulo por Barbosa et al. (2003).

\section{Resultados e Discussão}

A taxa geral de sobrevivência das 2.424 plântulas pertencentes a 110 espécies arbustivo-arbóreas foi de 69\% (1.675 indivíduos e 108 espécies sobreviventes), entretanto quando Protium spruceanum é desconsiderada da análise, a taxa de sobrevivência sobe para 75,4\% (Tabela 1).

Do total de espécies, 52 alcançaram $100 \%$ de sobrevivência em viveiro, entretanto 36 delas tiveram menos de cinco indivíduos transplantados e, portanto, os resultados de sobrevivência em viveiro dessas espécies devem ser vistos com cautela. De maneira contrária, apenas duas espécies, Inga marginata (um indivíduo) e Indet sp9 (dois indivíduos), tiveram $100 \%$ de mortalidade. Do total de indivíduos transferidos do remanescente para o viveiro, 27 morreram antes da identificação (Tabela 2).

Das 22 espécies com pelo menos 20 indivíduos transferidos para o viveiro, 13 apresentaram taxas de sobrevivência superiores a $75 \%$ e destas, somente Esenbeckia febrifuga teve $100 \%$ de sobrevivência. Em cinco espécies, as taxas de sobrevivência ficaram entre 50 e $75 \%$ e apenas três apresentaram taxa de sobrevivência inferior a 50\% (Tabela 2). Das 18 famílias com mais de 20 indivíduos, dez se destacaram, com taxa superior a $75 \%$ de sobrevivência, cinco apresentaram

Tabela 1. Número de indivíduos (N) e taxa de sobrevivência em viveiro (S) dos conjuntos de plântulas analisados nas diferentes classes de alturas $^{(1)}$.

\begin{tabular}{|c|c|c|c|c|c|c|c|c|}
\hline \multirow[t]{2}{*}{ Conjunto de indivíduos considerados } & \multicolumn{2}{|c|}{$\begin{array}{c}\text { Classe I } \\
\text { (até } 10 \mathrm{~cm} \text { ) }\end{array}$} & \multicolumn{2}{|c|}{$\begin{array}{c}\text { Classe II } \\
(11-20 \mathrm{~cm})\end{array}$} & \multicolumn{2}{|c|}{$\begin{array}{c}\text { Classe III } \\
(21-30 \mathrm{~cm})\end{array}$} & \multicolumn{2}{|c|}{ Geral } \\
\hline & $\mathrm{N}$ & $\mathrm{S}(\%)$ & $\mathrm{N}$ & $\mathrm{S}(\%)$ & $\mathrm{N}$ & $\mathrm{S}(\%)$ & $\mathrm{N}$ & $\mathrm{S}(\%)$ \\
\hline Todos & 1.275 & $66,6 b$ & 867 & $73,7 \mathrm{a}$ & 282 & $66,3 b$ & 2.424 & 69,0 \\
\hline Todos (sem Protium spruceanum) & 547 & $76,1 \mathrm{a}$ & 720 & $78,5 \mathrm{a}$ & 259 & $65,6 b$ & 1.526 & 75,4 \\
\hline Protium spruceanum & 728 & $59,5 \mathrm{a}$ & 147 & $50,3 \mathrm{a}$ & 23 & $73,9 \mathrm{a}$ & 898 & 58,4 \\
\hline Pioneiras & 179 & $82,7 \mathrm{a}$ & 287 & $86,0 \mathrm{a}$ & 86 & $76,7 \mathrm{a}$ & 552 & 83,5 \\
\hline Não pioneiras & 205 & $82,4 a$ & 325 & $78,8 \mathrm{a}$ & 144 & $61,1 b$ & 674 & 76,1 \\
\hline
\end{tabular}

(1)Letras diferentes nas linhas representam diferenças significativas nas taxas de sobrevivência entre as classes de altura (qui-quadrado, 5\% de probabilidade). 
Tabela 2. Número de indivíduos $(\mathrm{N})$ e taxa de sobrevivência em viveiro (S) das plântulas transplantadas para o viveiro por espécie, nas diferentes classes de altura.

\begin{tabular}{|c|c|c|c|c|c|c|c|c|c|c|}
\hline \multirow[t]{2}{*}{ Espécie } & \multirow[t]{2}{*}{ Nome vulgar } & \multirow[t]{2}{*}{$\mathrm{ES}^{(1)}$} & \multicolumn{2}{|c|}{$\begin{array}{c}\text { Classe I } \\
\text { (até } 10 \mathrm{~cm} \text { ) }\end{array}$} & \multicolumn{2}{|c|}{$\begin{array}{c}\text { Classe II } \\
(11-20 \mathrm{~cm})\end{array}$} & \multicolumn{2}{|c|}{$\begin{array}{c}\text { Classe III } \\
(21-30 \mathrm{~cm}) \mathrm{N} \\
\end{array}$} & \multicolumn{2}{|c|}{ Geral } \\
\hline & & & $\mathrm{N}$ & $\mathrm{S}(\%)$ & $\mathrm{N}$ & $\mathrm{S}(\%)$ & $\mathrm{N}$ & $\mathrm{S}(\%)$ & $\mathrm{N}$ & $\mathrm{S}(\%)$ \\
\hline Actinostemon communis $^{(2)}$ & Laranjeira-brava & NP & 6 & 100,0 & 6 & 100,0 & 2 & 100,0 & 14 & 100,0 \\
\hline Albizia polycephala & - & $\mathrm{NC}$ & - & - & 1 & 100,0 & - & - & 1 & 100,0 \\
\hline Alchornea triplinervia & Tapiá & $\mathrm{P}$ & 1 & 0,0 & 2 & 50,0 & 1 & 100,0 & 4 & 50,0 \\
\hline Allophylus edulis & Chal-chal & $\mathrm{P}$ & - & - & 3 & 100,0 & - & - & 3 & 100,0 \\
\hline Amaioua intermedia & Carvoeiro & NP & 1 & 0,0 & 2 & 100,0 & - & - & 3 & 66,7 \\
\hline Andira fraxinifolia & Angelim & $\mathrm{P}$ & 1 & 0,0 & 2 & 50,0 & - & - & 3 & 33,3 \\
\hline Aspidosperma subincanum $^{(2)}$ & Peroba-do-cerrado & NP & 2 & 100,0 & - & - & - & - & 2 & 100,0 \\
\hline Balfourodendron riedelianum & Pau-marfim & NP & 2 & 100,0 & 1 & 100,0 & - & - & 3 & 100,0 \\
\hline Bauhinia longifolia & Pata-de-vaca & $\mathrm{NC}$ & 2 & 100,0 & 6 & 83,3 & 2 & 50,0 & 10 & 80,0 \\
\hline Calliandra tweediei & Caliandra & $\mathrm{P}$ & 1 & 100,0 & - & - & - & - & 1 & 100,0 \\
\hline Calophyllum brasiliense & Guanandi & $\mathrm{NC}$ & - & - & 5 & 20,0 & 1 & 100,0 & 6 & 33,3 \\
\hline Campomanesia cf. xanthocarpa & Guabiroba & NP & 2 & 100,0 & 1 & 100,0 & 1 & 100,0 & 4 & 100,0 \\
\hline Casearia decandra(2) & Guaçatonga & NP & 3 & 100,0 & 2 & 100,0 & 2 & 100,0 & 7 & 100,0 \\
\hline Casearia sylvestris & Guaçatonga & $\mathrm{P}$ & - & - & 1 & 100,0 & - & - & 1 & 100,0 \\
\hline Cedrela fissilis & Cedro-rosa & NP & 1 & 100,0 & 2 & 50,0 & - & - & 3 & 66,7 \\
\hline Centrolobium tomentosum & Araribá & $\mathrm{P}$ & 1 & 100,0 & 5 & 80,0 & 3 & 100,0 & 9 & 88,9 \\
\hline Cestrum cf. sendtnerianum & - & $\mathrm{P}$ & 2 & 100,0 & 1 & 100,0 & 2 & 100,0 & 5 & 100,0 \\
\hline Cestrum schlechtendalii $^{(2)}$ & - & $\mathrm{NC}$ & 4 & 100,0 & - & - & - & - & 4 & 100,0 \\
\hline Chomelia obtusa $^{(2)}$ & - & NP & 1 & 100,0 & 2 & 100,0 & 2 & 100,0 & 5 & 100,0 \\
\hline Coccoloba cordata ${ }^{(2)}$ & - & $\mathrm{NC}$ & 3 & 66,7 & - & - & - & - & 3 & 66,7 \\
\hline Copaifera langsdorffii & Copaíba & NP & 1 & 0,0 & 10 & 40,0 & 12 & 8,3 & 23 & 21,7 \\
\hline Cordia sellowiana & Jurutê & $\mathrm{P}$ & - & - & 3 & 100,0 & 2 & 100,0 & 5 & 100,0 \\
\hline Croton floribundus & Capixingui & $\mathrm{P}$ & 17 & 82,4 & 29 & 93,1 & 9 & 100,0 & 55 & 90,9 \\
\hline Cupania tenuivalvis(2) & Camboatã & NP & 9 & 44,4 & 20 & 65,0 & 1 & 0,0 & 30 & 56,7 \\
\hline Dalbergia frutescens ${ }^{(2)}$ & - & $\mathrm{NC}$ & 49 & 98,0 & 13 & 92,3 & 2 & 50,0 & 64 & 95,3 \\
\hline Diospyros inconstans & Caqui-do-mato & NP & - & - & 1 & 100,0 & - & - & 1 & 100,0 \\
\hline Endlicheria paniculata $^{(2)}$ & Canela-do-brejo & NP & - & - & - & - & 1 & 100,0 & 1 & 100,0 \\
\hline Erythroxylum cuneifolium (2) & - & NP & - & - & 1 & 100,0 & 4 & 100,0 & 5 & 100,0 \\
\hline Esenbeckia febrifuga ${ }^{(2)}$ & Mamoninha-do-mato & NP & 3 & 100,0 & 12 & 100,0 & 8 & 100,0 & 23 & 100,0 \\
\hline Esenbeckia grandiflora & Guaxupita & NP & - & - & 4 & 100,0 & 1 & 100,0 & 5 & 100,0 \\
\hline Eugenia cf. hyemalis & - & NP & - & - & 1 & 100,0 & - & - & 1 & 100,0 \\
\hline Eugenia florida & - & NP & - & - & 1 & 100,0 & - & - & 1 & 100,0 \\
\hline Eugenia ligustrina $^{(2)}$ & - & NP & 37 & 94,6 & 31 & 100,0 & 3 & 100,0 & 71 & 97,2 \\
\hline Eugenia pluriflora ${ }^{(2)}$ & - & NP & 13 & 92,3 & 6 & 100,0 & - & - & 19 & 94,7 \\
\hline Euterpe edulis & Palmito-juçara & NP & - & - & 9 & 66,7 & 8 & 50,0 & 17 & 58,8 \\
\hline Faramea montevidensis ${ }^{(2)}$ & - & NP & 6 & 33,3 & 5 & 20,0 & - & - & 11 & 27,3 \\
\hline Geonoma brevispatha ${ }^{(2)}$ & Guaricanga-do-brejo & NP & - & - & 2 & 50,0 & 2 & 100,0 & 4 & 75,0 \\
\hline Gochnatia polymorpha & Cambará & $\mathrm{NC}$ & 2 & 100,0 & 1 & 100,0 & 1 & 100,0 & 4 & 100,0 \\
\hline Gomidesia affinis & Perta-guela & NP & 12 & 91,7 & 11 & 72,7 & 3 & 66,7 & 26 & 80,8 \\
\hline Guapira hirsuta & Maria-mole & $\mathrm{P}$ & 1 & 100,0 & 12 & 100,0 & 6 & 100,0 & 19 & 100,0 \\
\hline Guatteria nigrescens $^{(2)}$ & Pindaíba-preta & NP & 1 & 100,0 & - & - & 2 & 100,0 & 3 & 100,0 \\
\hline Indet sp1 & - & $\mathrm{NC}$ & - & - & 1 & 100,0 & - & - & 1 & 100,0 \\
\hline Indet $\mathrm{sp} 2$ & - & $\mathrm{NC}$ & 4 & 75,0 & - & - & - & - & 4 & 75,0 \\
\hline Indet sp3 & - & $\mathrm{NC}$ & - & - & 1 & 100,0 & - & - & 1 & 100,0 \\
\hline Indet sp4 & - & $\mathrm{NC}$ & 1 & 100,0 & - & - & - & - & 1 & 100,0 \\
\hline Indet sp5 & - & $\mathrm{NC}$ & - & - & - & - & 1 & 100,0 & 1 & 100,0 \\
\hline Indet sp6 & - & $\mathrm{NC}$ & - & - & 2 & 100,0 & - & - & 2 & 100,0 \\
\hline Indet sp7 & - & $\mathrm{NC}$ & 1 & 100,0 & - & - & - & - & 1 & 100,0 \\
\hline Indet $\mathrm{sp} 8$ & - & $\mathrm{NC}$ & - & - & 1 & 100,0 & - & - & 1 & 100,0 \\
\hline Indet sp9 & - & $\mathrm{NC}$ & - & - & - & - & 2 & 0,0 & 2 & 0,0 \\
\hline Indet sp10 & - & $\mathrm{NC}$ & 4 & 50,0 & 2 & 50,0 & - & - & 6 & 50,0 \\
\hline Inga marginata & Ingá & $\mathrm{P}$ & - & - & - & - & 1 & 0,0 & 1 & 0,0 \\
\hline Inga vera & Ingá & $\mathrm{P}$ & 9 & 100,0 & 19 & 78,9 & 1 & 0,0 & 29 & 82,8 \\
\hline Lacistema hasslerianum & - & NP & 4 & 100,0 & 4 & 100,0 & 2 & 100,0 & 10 & 100,0 \\
\hline
\end{tabular}


Tabela 2. Continuação.

\begin{tabular}{|c|c|c|c|c|c|c|c|c|c|c|}
\hline \multirow[t]{2}{*}{ Espécie } & \multirow[t]{2}{*}{ Nome vulgar } & \multirow[t]{2}{*}{$\mathrm{ES}^{(1)}$} & \multicolumn{2}{|c|}{$\begin{array}{c}\text { Classe I } \\
\text { (até } 10 \mathrm{~cm} \text { ) }\end{array}$} & \multicolumn{2}{|c|}{$\begin{array}{c}\text { Classe II } \\
(11-20 \mathrm{~cm}) \\
\end{array}$} & \multicolumn{2}{|c|}{$\begin{array}{c}\text { Classe III } \\
(21-30 \mathrm{~cm})\end{array}$} & \multicolumn{2}{|c|}{ Geral } \\
\hline & & & $\mathrm{N}$ & $\mathrm{S}(\%)$ & $\mathrm{N}$ & $\mathrm{S}(\%)$ & $\mathrm{N}$ & $\mathrm{S}(\%)$ & $\mathrm{N}$ & $\mathrm{S}(\%)$ \\
\hline Lafoensia pacari & Dedaleiro & $\mathrm{P}$ & 1 & 100,0 & - & - & - & - & 1 & 100,0 \\
\hline Leandra scabra ${ }^{(2)}$ & - & $\mathrm{NC}$ & 1 & 100,0 & 6 & 50,0 & - & - & 7 & 57,1 \\
\hline Leandra sp. & - & $\mathrm{NC}$ & - & - & 1 & 100,0 & - & - & 1 & 100,0 \\
\hline Lonchocarpus cultratus & Embira-de-sapo & $\mathrm{P}$ & 2 & 50,0 & 2 & 100,0 & - & - & 4 & 75,0 \\
\hline Machaerium aculeatum & Bico-de-pato & $\mathrm{P}$ & 7 & 100,0 & 8 & 100,0 & - & - & 15 & 100,0 \\
\hline Machaerium brasiliense & Jacarandá & $\mathrm{P}$ & - & - & 3 & 100,0 & 3 & 100,0 & 6 & 100,0 \\
\hline Machaerium stipitatum & Sapuvinha & $\mathrm{P}$ & 2 & 100,0 & 5 & 100,0 & - & - & 7 & 100,0 \\
\hline Maprounea guianensis ${ }^{(2)}$ & Marmeleiro-do-campo & $\mathrm{P}$ & 5 & 60,0 & - & - & 1 & 0,0 & 6 & 50,0 \\
\hline Matayba elaeagnoides & Miguel-pintado & $\mathrm{P}$ & 6 & 83,3 & 34 & 70,6 & 8 & 50,0 & 48 & 68,8 \\
\hline Maytenus aquifolia(2) & Espinheira-santa & NP & - & - & 1 & 100,0 & - & - & 1 & 100,0 \\
\hline Maytenus salicifolia(2) & Cafezinho & NP & 31 & 83,9 & 72 & 66,7 & 39 & 43,6 & 142 & 64,1 \\
\hline Miconia ligustroides $^{(2)}$ & - & $\mathrm{P}$ & 3 & 33,3 & 2 & 100,0 & 2 & 50,0 & 7 & 57,1 \\
\hline Mollinedia schottiana $(2)$ & - & NP & 1 & 100,0 & 1 & 100,0 & 1 & 100,0 & 3 & 100,0 \\
\hline Mortas antes da morfo-identificação & - & $\mathrm{NC}$ & 15 & 0,0 & 7 & 0,0 & 5 & 0,0 & 27 & 0,0 \\
\hline Myrcia cf. hartwegiana & Cambuí & NP & 3 & 66,7 & - & - & 2 & 100,0 & 5 & 80,0 \\
\hline Myrcia fallax & Guamirim & $\mathrm{P}$ & 16 & 100,0 & 38 & 97,4 & 12 & 75,0 & 66 & 93,9 \\
\hline Myrcia guianensis ${ }^{(2)}$ & Cambuí & NP & 4 & 75,0 & 7 & 100,0 & 6 & 100,0 & 17 & 94,1 \\
\hline Myrcia multiflora & Cambuí & NP & 2 & 100,0 & 11 & 100,0 & 4 & 100,0 & 17 & 100,0 \\
\hline Myrciaria cf. tenella & - & NP & 4 & 100,0 & 2 & 100,0 & 3 & 66,7 & 9 & 88,9 \\
\hline Myrciaria floribunda ${ }^{(2)}$ & - & NP & 2 & 100,0 & 1 & 0,0 & 4 & 25,0 & 7 & 42,9 \\
\hline Myrtaceae sp8 & - & $\mathrm{NC}$ & - & - & 2 & 50,0 & 2 & 50,0 & 4 & 50,0 \\
\hline Myrtaceae sp9 & - & $\mathrm{NC}$ & 1 & 100,0 & - & - & - & - & 1 & 100,0 \\
\hline Nectandra cf. grandiflora & Canela-amarela & NP & 3 & 100,0 & 14 & 71,4 & 5 & 80,0 & 22 & 77,3 \\
\hline Ocotea cf. velutina & Canelão-amarelo & $\mathrm{NC}$ & 9 & 66,7 & 4 & 100,0 & 6 & 83,3 & 19 & 78,9 \\
\hline Ocotea corymbosa ${ }^{(2)}$ & Canela-fedorenta & $\mathrm{P}$ & - & - & 1 & 100,0 & 1 & 100,0 & 2 & 100,0 \\
\hline Palicourea marcgravii ${ }^{(2)}$ & Erva-de-rato & $\mathrm{NC}$ & 53 & 35,8 & 27 & 33,3 & 6 & 66,7 & 86 & 37,2 \\
\hline Pera glabrata & Tabocúva & NP & 3 & 100,0 & 6 & 83,3 & - & - & 9 & 88,9 \\
\hline Persea pyrifolia & Abacateiro-do-mato & NP & - & - & 1 & 100,0 & - & - & 1 & 100,0 \\
\hline Piper gaudichaudianum ${ }^{(2)}$ & Falso-jaborandi & $\mathrm{NC}$ & - & - & 3 & 100,0 & - & - & 3 & 100,0 \\
\hline Piptadenia gonoacantha & Pau-jacaré & $\mathrm{P}$ & - & - & 3 & 66,7 & 2 & 50,0 & 5 & 60,0 \\
\hline Protium spruceanum ${ }^{(2)}$ & Almecegueiro & $\mathrm{P}$ & 728 & 59,5 & 147 & 50,3 & 23 & 73,9 & 898 & 58,4 \\
\hline Prunus myrtifolia & Pessegueiro-bravo & $\mathrm{P}$ & 2 & 100,0 & 3 & 100,0 & 2 & 100,0 & 7 & 100,0 \\
\hline Psychotria cf. carthagenensis & - & NP & 1 & 100,0 & - & - & - & - & 1 & 100,0 \\
\hline Psychotria leiocarpa ${ }^{(2)}$ & - & NP & 2 & 100,0 & 1 & 100,0 & - & - & 3 & 100,0 \\
\hline Psychotria sp. & - & $\mathrm{NC}$ & - & - & 4 & 50,0 & 1 & 100,0 & 5 & 60,0 \\
\hline Psychotria vellosiana ${ }^{(2)}$ & - & NP & 25 & 60,0 & 23 & 69,6 & 5 & 60,0 & 53 & 64,2 \\
\hline Rapanea cf. umbellata & Capororocão & $\mathrm{P}$ & 31 & 83,9 & 16 & 62,5 & 2 & 100,0 & 49 & 77,6 \\
\hline Rapanea ferruginea & Capororoca & $\mathrm{P}$ & 4 & 75,0 & 10 & 90,0 & 1 & 0,0 & 15 & 80,0 \\
\hline Roupala brasiliensis & Carne-de-vaca & NP & 4 & 50,0 & 20 & 80,0 & 15 & 40,0 & 39 & 61,5 \\
\hline Rubiaceae sp1 & - & NP & 3 & 100,0 & 2 & 100,0 & - & - & 5 & 100,0 \\
\hline Sebastiania commersoniana & Branquilho & NP & 6 & 83,3 & 5 & 100,0 & 1 & 100,0 & 12 & 91,7 \\
\hline Siparuna cujabana ${ }^{(2)}$ & Figueirinha & NP & 9 & 33,3 & 16 & 50,0 & - & - & 25 & 44,0 \\
\hline Siparuna guianensis ${ }^{(2)}$ & Limão-bravo & NP & 1 & 100,0 & - & - & - & - & 1 & 100,0 \\
\hline Siphoneugenia aff. widgreniana & - & NP & 3 & 66,7 & 8 & 87,5 & 1 & 0,0 & 12 & 75,0 \\
\hline Sloanea monosperma $(2)$ & - & NP & 1 & 100,0 & 8 & 87,5 & 2 & 100,0 & 11 & 90,9 \\
\hline Styrax pohlii & Benjoeiro & $\mathrm{NC}$ & - & - & - & - & 1 & 100,0 & 1 & 100,0 \\
\hline Syagrus romanzoffiana & Jerivá & $\mathrm{P}$ & 3 & 66,7 & 2 & 100,0 & - & - & 5 & 80,0 \\
\hline Symplocos tenuifolia ${ }^{(2)}$ & - & $\mathrm{NC}$ & - & - & 5 & 100,0 & 15 & 86,7 & 20 & 90,0 \\
\hline Tabernaemontana hystrix & Leiteiro & $\mathrm{P}$ & - & - & 1 & 100,0 & - & - & 1 & 100,0 \\
\hline Tapirira guianensis & Peito-de-pombo & $\mathrm{P}$ & 27 & 81,5 & 29 & 89,7 & 1 & 100,0 & 57 & 86,0 \\
\hline Terminalia triflora & Capitão-do-campo & $\mathrm{NC}$ & 27 & 77,8 & 20 & 85,0 & 3 & 100,0 & 50 & 82,0 \\
\hline Tibouchina sellowiana ${ }^{(2)}$ & Manacá & $\mathrm{NC}$ & 2 & 100,0 & 2 & 100,0 & - & - & 4 & 100,0 \\
\hline 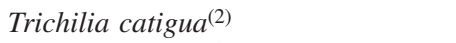 & Catiguá & NP & 2 & 100,0 & 6 & 83,3 & - & - & 8 & 87,5 \\
\hline Trichilia elegans ${ }^{(2)}$ & Catiguá & NP & - & - & 2 & 100,0 & 1 & 100,0 & 3 & 100,0 \\
\hline Trichilia pallida ${ }^{(2)}$ & Catiguá & $\mathrm{P}$ & 10 & 80,0 & 23 & 91,3 & 4 & 50,0 & 37 & 83,8 \\
\hline Vochysia tucanorum $^{(2)}$ & Pau-tucano & $\mathrm{P}$ & - & - & 2 & 50,0 & 3 & 0,0 & 5 & 20,0 \\
\hline Zanthoxylum rhoifolium & Mamica-de-porca & $\mathrm{P}$ & 3 & 66,7 & 6 & 50,0 & 1 & 100,0 & 10 & 60,0 \\
\hline
\end{tabular}

${ }^{(1)}$ ES, estágio sucessional; NC, não caracterizada; NP, não pioneira; P, pioneira. ${ }^{(2)}$ Espécie não encontrada nos 30 principais viveiros de espécies nativas do Estado de São Paulo (Barbosa et al., 2003). 
taxas de sobrevivência dos indivíduos entre 50 e $75 \%$ e apenas duas revelaram taxa inferior a $50 \%$ de sobrevivência (Tabela 3 ).

Sob condições naturais do sub-bosque florestal, a sobrevivência e a capacidade das plântulas de se adaptarem às mudanças do ambiente dependem de uma complexa interação entre seus atributos morfológicos e fisiológicos (Fenner, 1987). Tais atributos incluem principalmente área foliar, biomassa, disposição das folhas e ramos, taxas fotossintética e respiratória e eficiência no uso da água (Garwood, 1996). A sobrevivência e a capacidade de desenvolvimento destes indivíduos, quando transplantados para viveiro de produção de mudas, também seria afetada pelos mesmos aspectos. É evidente que as espécies apresentam características morfológicas e fisiológicas intrínsecas e muito específicas, portanto, justifica-se a variação nas taxas de sobrevivência em viveiro para cada espécie, conforme resultados deste estudo.

A taxa de sobrevivência foi significativamente superior nas espécies pioneiras, quando comparadas com as não pioneiras $\left(\chi^{2}=9,73 ; p=0,0018\right)$. O comportamento diferenciado de pioneiras e não pioneiras em viveiro é conhecido na produção de mudas de espécies nativas, quando propagadas por sementes (Gonçalves et al., 2000) e parece ser também evidente quando as mudas são obtidas por meio do transplante da regeneração natural.

Plântulas não pioneiras germinam e permanecem sob o dossel florestal, em locais sombreados, onde a luminosidade é bastante reduzida, a umidade do ar é mais alta e a temperatura mais amena (Swaine \& Whitmore, 1988). Assim, sofrem maior impacto quando transferidas para viveiro a pleno sol ou mesmo com certo grau de sombreamento, principalmente por não suportarem os aumentos na radiação solar, na temperatura e na umidade. No entanto, neste trabalho há várias exceções de espécies não pioneiras que apresentaram altos índices de sobrevivência na transferência ao viveiro, tais como Actinostemon communis, Esenbeckia febrifuga, Lacistema hasslerianum e muitas espécies da família Myrtaceae, de forma que tais impactos no transplante não se configuram como entraves à produção de mudas de espécies não pioneiras por meio da transferência da regeneração natural para viveiro.

Quando consideradas todas as plântulas transplantadas, a classe de altura II (11-20 cm de altura) apresenta a melhor taxa de sobrevivência (Tabela 1).
No entanto, em espécies não pioneiras, não há diferenças de sobrevivência entre as classes II (11-20 cm de altura) e III (21-30 cm de altura) e nas pioneiras não há diferenças na sobrevivência entre nenhuma das classes de altura.

Para as espécies, a classe de altura com a melhor taxa de sobrevivência foi variável. Portanto, nos casos em que a utilização dessa técnica visa ao transplante para viveiro, apenas de espécies pré-selecionadas, seria interessante considerar não a taxa de sobrevivência geral por classe de altura, mas a taxa de sobrevivência da espécie em questão por classe de altura.

Além disso, na utilização dessa prática visando à produção de mudas, deve-se considerar também a quantidade de plântulas disponíveis por classe de altura, na área de coleta. Salvo poucas exceções, espécies arbustivo-arbóreas tropicais apresentam número consideravelmente maior de indivíduos nas fases iniciais de vida, com decréscimo à medida que a idade aumenta (Fenner, 1987). Uma espécie pode apresentar maior taxa de sobrevivência na classe III, mas por haver menor disponibilidade de plântulas nessa classe, o número final de indivíduos sobreviventes no viveiro se torna maior nas classes I e II. Croton floribundus, por exemplo, embora apresente as melhores taxas de sobrevivência na classe III $(21-30 \mathrm{~cm}$ de altura), o número de indivíduos coletados e levados para viveiro nessa classe é bastante inferior ao das demais classes, de forma que, mesmo sob taxas inferiores de sobrevivência, ao final das avaliações, o número de indivíduos sobreviventes nas classes de altura I (até $10 \mathrm{~cm})$ e II $(11-20 \mathrm{~cm})$ foi maior que o da classe III $(21-30 \mathrm{~cm})$.

As taxas de mortalidade e a porcentagem dos indivíduos sobreviventes que emitiram folhas foram maiores na primeira avaliação, realizada três meses após o transplante, diminuindo nas avaliações seguintes (Tabela 4). Ao analisar estes parâmetros para as diferentes classes de altura, foi observada tendência da classe I (até $10 \mathrm{~cm}$ ) apresentar maiores taxa de mortalidade e porcentagem de indivíduos sobreviventes rebrotados três meses após o transplante e, também das classes II e III apresentarem menores diferenças nas taxas de mortalidade e porcentagem de indivíduos sobreviventes rebrotados entre a avaliação inicial e as avaliações subseqüentes. Assim, indivíduos de menor porte respondem de forma mais rápida ao transplante, ou com rebrota e emissão de folhas novas, ou com a morte. Por sua vez, conforme constatado neste 
trabalho, indivíduos maiores podem permanecer por um tempo num estado de latência, murchando e perdendo suas folhas, sem rebrotar ou emitir folhas novas, mas ao mesmo tempo sem dar indícios de estar morto. Porém, em todas as classes de altura, foi evidente que o período inicial após o transplante, representado pelos primeiros três meses após a transferência, é o período mais crítico para a sobrevivência das plântulas. Uma vez ultrapassada essa etapa, as chances do indivíduo sobreviver e se desenvolver aumentam.

Ao final das avaliações, o viveiro contava com mudas de 108 espécies arbustivo-arbóreas, número superior aos

Tabela 3. Número de indivíduos (N) e taxa de sobrevivência em viveiro (S) por família transplantada para o viveiro, nas diferentes classes de altura.

\begin{tabular}{|c|c|c|c|c|c|c|c|c|c|}
\hline \multirow[t]{2}{*}{ Família } & \multirow[t]{2}{*}{$\begin{array}{c}\text { № de } \\
\text { espécies }\end{array}$} & \multicolumn{2}{|c|}{$\begin{array}{c}\text { Classe I } \\
\text { (até } 10 \mathrm{~cm} \text { ) }\end{array}$} & \multicolumn{2}{|c|}{$\begin{array}{c}\text { Classe II } \\
(11-20 \mathrm{~cm})\end{array}$} & \multicolumn{2}{|c|}{$\begin{array}{l}\text { Classe III } \\
(21-30 \mathrm{~cm})\end{array}$} & \multicolumn{2}{|c|}{ Geral } \\
\hline & & $\mathrm{N}$ & $\mathrm{S}(\%)$ & $\mathrm{N}$ & $\mathrm{S}(\%)$ & $\mathrm{N}$ & $\mathrm{S}(\%)$ & $\mathrm{N}$ & $\mathrm{S}(\%)$ \\
\hline Anacardiaceae & 1 & 27 & 77,8 & 20 & 85,0 & 3 & 100,0 & 50 & 82,0 \\
\hline Annonaceae & 1 & 1 & 100,0 & 0 & 0,0 & 2 & 100,0 & 3 & 100,0 \\
\hline Apocynaceae & 2 & 29 & 82,8 & 29 & 89,7 & 1 & 100,0 & 59 & 86,4 \\
\hline Arecaceae & 3 & 0 & 0,0 & 16 & 75,0 & 25 & 76,0 & 41 & 75,6 \\
\hline Asteraceae & 1 & 2 & 100,0 & 1 & 100,0 & 1 & 100,0 & 4 & 100,0 \\
\hline Boraginaceae & 1 & 0 & 0,0 & 3 & 100,0 & 2 & 100,0 & 5 & 100,0 \\
\hline Burseraceae & 1 & 728 & 59,5 & 147 & 50,3 & 23 & 73,9 & 898 & 58,4 \\
\hline Celastraceae & 2 & 31 & 83,9 & 73 & 67,1 & 39 & 43,6 & 143 & 64,3 \\
\hline Clusiaceae & 1 & 0 & 0,0 & 5 & 20,0 & 1 & 100,0 & 6 & 33,3 \\
\hline Ebenaceae & 1 & 0 & 0,0 & 1 & 100,0 & 0 & 0,0 & 1 & 100,0 \\
\hline Elaeocarpaceae & 1 & 0 & 0,0 & 0 & 0,0 & 1 & 100,0 & 1 & 100,0 \\
\hline Erythroxylaceae & 1 & 0 & 0,0 & 1 & 100,0 & 4 & 100,0 & 5 & 100,0 \\
\hline Euphorbiaceae & 6 & 38 & 81,6 & 48 & 91,7 & 14 & 92,9 & 100 & 88,0 \\
\hline Fabaceae-Caesalpinioideae & 1 & 1 & 0,0 & 10 & 40,0 & 12 & 8,3 & 23 & 21,7 \\
\hline Fabaceae-Cercidae & 1 & 2 & 100,0 & 6 & 83,3 & 2 & 50,0 & 10 & 80,0 \\
\hline Fabaceae-Mimosoideae & 5 & 10 & 100,0 & 23 & 78,3 & 4 & 25,0 & 37 & 78,4 \\
\hline Fabaceae-Papilionoideae & 7 & 62 & 95,2 & 38 & 92,1 & 8 & 87,5 & 108 & 93,5 \\
\hline Lacistemaceae & 1 & 4 & 100,0 & 4 & 100,0 & 2 & 100,0 & 10 & 100,0 \\
\hline Lauraceae & 5 & 12 & 75,0 & 20 & 80,0 & 13 & 84,6 & 45 & 80,0 \\
\hline Lythraceae & 1 & 1 & 100,0 & 0 & 0,0 & 0 & 0,0 & 1 & 100,0 \\
\hline Melastomataceae & 4 & 6 & 66,7 & 11 & 72,7 & 2 & 50,0 & 19 & 68,4 \\
\hline Meliaceae & 4 & 13 & 84,6 & 33 & 87,9 & 5 & 60,0 & 51 & 84,3 \\
\hline Monimiaceae & 1 & 1 & 100,0 & 1 & 100,0 & 1 & 100,0 & 3 & 100,0 \\
\hline Morta antes da morfo-identificação & - & 15 & 0,0 & 7 & 0,0 & 5 & 0,0 & 27 & 0,0 \\
\hline Myrsinaceae & 2 & 35 & 82,9 & 26 & 73,1 & 3 & 66,7 & 64 & 78,1 \\
\hline Myrtaceae & 15 & 97 & 93,8 & 120 & 94,2 & 42 & 78,6 & 259 & 91,5 \\
\hline Nyctaginaceae & 1 & 1 & 100,0 & 12 & 100,0 & 6 & 100,0 & 19 & 100,0 \\
\hline Piperaceae & 1 & 0 & 0,0 & 3 & 100,0 & 0 & 0,0 & 3 & 100,0 \\
\hline Polygalaceae & 1 & 9 & 33,3 & 16 & 50,0 & 0 & 0,0 & 25 & 44,0 \\
\hline Polygonaceae & 1 & 3 & 66,7 & 0 & 0,0 & 0 & 0,0 & 3 & 66,7 \\
\hline Proteaceae & 1 & 4 & 50,0 & 20 & 80,0 & 15 & 40,0 & 39 & 61,5 \\
\hline Rosaceae & 1 & 2 & 100,0 & 3 & 100,0 & 2 & 100,0 & 7 & 100,0 \\
\hline Rubiaceae & 9 & 92 & 46,7 & 66 & 53,0 & 14 & 71,4 & 172 & 51,2 \\
\hline Rutaceae & 4 & 8 & 87,5 & 23 & 87,0 & 10 & 100,0 & 41 & 90,2 \\
\hline Salicaceae & 2 & 3 & 100,0 & 3 & 100,0 & 2 & 100,0 & 8 & 100,0 \\
\hline Sapindaceae & 3 & 15 & 60,0 & 57 & 70,2 & 9 & 44,4 & 81 & 65,4 \\
\hline Siparunaceae & 2 & 4 & 75,0 & 8 & 87,5 & 1 & 0,0 & 13 & 76,9 \\
\hline Solanaceae & 2 & 6 & 100,0 & 1 & 100,0 & 2 & 100,0 & 9 & 100,0 \\
\hline Styracaceae & 1 & 3 & 66,7 & 2 & 100,0 & 0 & 0,0 & 5 & 80,0 \\
\hline Symplocaceae & 1 & 0 & 0,0 & 1 & 100,0 & 0 & 0,0 & 1 & 100,0 \\
\hline Vochysiaceae & 1 & 0 & 0,0 & 2 & 50,0 & 3 & 0,0 & 5 & 20,0 \\
\hline Indeterminada & 10 & 10 & 70,0 & 7 & 85,7 & 3 & 33,3 & 20 & 70,0 \\
\hline
\end{tabular}


comumente encontrados nos viveiros de espécies nativas e nos plantios de restauração de áreas degradadas. Esses números são maiores até mesmo que os exigidos pela legislação do Estado de São Paulo, que constam na resolução SMA-58 de 2006 (São Paulo, 2006), que estabelece número mínimo de 80 espécies nativas em plantios para fins de restauração.

Ao serem comparados os resultados deste trabalho com os da produção de mudas nativas no Estado de São Paulo (Barbosa et al., 2003), pode-se observar que muitas espécies transplantadas sequer são encontradas nos 30 principais viveiros do Estado (Tabela 2). Ao se considerar apenas as espécies identificadas que ao final do período de avaliações continham pelo menos dez indivíduos sobreviventes no viveiro, foi possível produzir mudas de 12 espécies arbustivo-arbóreas não encontradas nos viveiros analisados por Barbosa et al. (2003).

Muitas espécies transplantadas não são encontradas nos viveiros, provavelmente em função da dificuldade na coleta de sementes, ou mesmo pelo desconhecimento dos seus mecanismos fisiológicos e das tecnologias empregadas na germinação de suas sementes. Em muitas destas espécies, a transferência dos regenerantes para viveiro surge como alternativa simples e viável de produção de mudas, que elimina etapas difíceis de serem executadas, às vezes caras e desconhecidas como o beneficiamento, armazenamento e tratamento prégerminativo das sementes.

Tabela 4. Taxas de mortalidade e porcentagem de indivíduos sobreviventes rebrotados nas classes de altura, nos diferentes períodos de avaliação dos indivíduos transplantados de remanescente florestal para viveiro de produção de mudas.

\begin{tabular}{lccc}
\hline Classes de altura & $\begin{array}{c}\text { Meses } \\
\text { após } \\
\text { transplante }\end{array}$ & $\begin{array}{c}\text { Taxa de } \\
\text { mortalidade } \\
(\%)\end{array}$ & $\begin{array}{c}\text { Indivíduos } \\
\text { sobreviventes } \\
\text { rebrotados }(\%)\end{array}$ \\
\hline I (até $10 \mathrm{~cm})$ & $0-3$ & 24,39 & 78,68 \\
& $3-5$ & 3,29 & 17,90 \\
& $5-7$ & 2,90 & 2,24 \\
& $7-9$ & 2,82 & 1,18 \\
\hline II $(11-20 \mathrm{~cm})$ & $0-3$ & 15,11 & 66,20 \\
& $3-5$ & 3,92 & 21,28 \\
& $5-7$ & 4,15 & 9,23 \\
III $(21-30 \mathrm{~cm})$ & $7-9$ & 3,11 & 3,29 \\
& $0-3$ & 12,77 & 56,68 \\
& $3-5$ & 8,87 & 19,25 \\
& $5-7$ & 7,80 & 12,30 \\
& $7-9$ & 4,26 & 11,76 \\
\hline Geral & $0-3$ & 19,72 & 71,46 \\
& $3-5$ & 4,17 & 19,34 \\
& $5-7$ & 3,92 & 6,03 \\
& $7-9$ & 3,09 & 3,16 \\
\hline
\end{tabular}

Dessa forma, a transferência de plântulas de áreas naturais para viveiro é uma técnica vantajosa de produção de mudas, que complementa a produção de mudas por sementes e eleva a diversidade de espécies disponíveis nos viveiros. Não foram avaliados os impactos que a transferência dos regenerantes para viveiro pode gerar na dinâmica de regeneração da comunidade. Portanto, essa técnica deve ser utilizada de forma limitada e controlada, a não ser nos casos de remanescentes florestais que irreversivelmente serão desmatados com a autorização dos órgãos de licenciamento ambiental, como áreas de represamento para geração de energia, de construção e manutenção de estradas e de mineração, sendo nestes casos uma importante medida mitigadora dos impactos gerados.

\section{Conclusões}

1. A taxa de sobrevivência em viveiro de mudas de espécies nativas retiradas da regeneração natural é variável de acordo com a espécie, com o estágio sucessional e com a altura dos indivíduos transplantados.

2. Plântulas de espécies pioneiras, quando transferidas da regeneração natural, apresentam taxa de sobrevivência significativamente maior que plântulas de espécies não pioneiras.

3. A transferência de plântulas arbustivo-arbóreas de fragmentos florestais para viveiro é viável como técnica de produção de mudas de espécies nativas.

\section{Agradecimentos}

À Capes, pela bolsa de pós-graduação; à Eucatex Florestal S/A, pela área e infra-estrutura disponibilizadas.

\section{Referências}

ANGIOSPERM PHYLOGENY GROUP - APG. An update of the angiosperm phylogeny group classification for the orders and families of flowering plants: APG II. Botanical Journal of the Linnean Society, v.141, p.399-436, 2003.

AUER, C.G.; GRAÇA, M.C.E. Método de produção de mudas de canela-sassafrás a partir de mudas de regeneração natural. Boletim de Pesquisas Florestais, n.30/31, p.75-77, 1995.

BARBOSA, L.M.; BARBOSA, J.M.; BARBOSA, K.C.; POTOMATI, A.; MARTINS, S.E.; ASPERTI, L.M.; MELO, A.C.G.; CARRASCO, P.G.; CASTANHEIRA, S.A.; PILIACKAS, J.M.; CONTIERI, W.A.; MATTIOLI, D.S.; GUEDES, D.C.; SANTOS JÚNIOR, N.; SILVA, P.M.S.; PLAZA, A.P. Recuperação florestal com espécies nativas no Estado de 
São Paulo: pesquisas apontam mudanças necessárias. Florestar Estatístico, v.6, p.28-34, 2003.

BEIGUELMAN, B. Curso prático de bioestatística. 3.ed. Ribeirão Preto: Sociedade Brasileira de Genética, 1994. 244p.

CORVELLO, W.B.V. Utilização de mudas da regeneração natural em reflorestamentos com espécies nativas. 1983.105p. Dissertação (Mestrado) - Universidade Federal do Paraná, Curitiba.

FENNER, M. Seedlings. The New Phytologist, v.106, p.35-47, 1987. FERRETTI, A.R.; KAGEYAMA, P.Y.; ÁRBOCZ, G. de F.; SANTOS, J.D.; BARROS, M.I.A. de; LORZA, R.A.F.; OLIVEIRA, C. de. Classificação das espécies arbóreas em grupos ecológicos para revegetação com nativas no Estado de São Paulo. Florestar Estatístico, v.3, p.73-77,1995.

FONSECA, C.E.L da; RIBEIRO, J.F.; SOUZA, C.C. de; REZENDE, R.P.; BALBINO, V.K. Recuperação da vegetação de matas de galeria: estudos de caso no Distrito Federal e entorno. In: RIBEIRO, J.F.; FONSECA, C.E.L. da; SOUZA-SILVA, J.C. (Ed.). Caracterização e recuperação de matas de galeria. Planaltina: Embrapa Cerrados. 2001. p.815-870.

GARWOOD, N.C. Functional morphology of tropical tree seedlings. In: SWAINE, M.D. (Ed.). The ecology of tropical forest tree seedlings. Paris: UNESCO and Parthenon Publishing Group, 1996. p.59-129.

GONÇALVES, J.L.M.; SANTARELLI, E.D.; MORAES NETO, S.P. de; MANARA, M.P. Produção de mudas de espécies nativas: substrato, nutrição, sombreamento e fertilização. In: GONÇALVES, J.L.M.; BENEDETTI, V. (Ed.). Nutrição e fertilização florestal. Piracicaba: IPEF, 2000. p.309-350.

IVANAUSKAS, N.M.; RODRIGUES, R.R.; NAVE, A.A. Fitossociologia de um remanescente de floresta estacional semidecidual em Itatinga-SP, para fins de restauração de áreas degradadas. Revista Árvore, v.26, p.43-57, 2002.
NEMER, TG; de mudas da regeneração natural de espécies arbóreas três meses após o plantio em clareiras de diferentes tamanhos, MojuPA. Revista Árvore, v.26, p.217-221, 2002.

OLIVEIRA, L.M. de; DAVIDE, A.C.; CARVALHO, M.L.M. de. Avaliação de métodos para quebra da dormência e para a desinfestação de sementes de canafístula (Peltophorum dubium (Sprengel) Taubert. Revista Árvore, v.27, p.597-603, 2003.

RODRIGUES, R.R.; GANDOLFI, S. Conceitos, tendências e ações para recuperação de florestas ciliares. In: RODRIGUES, R.R.; LEITÃO FILHO, H.F. (Ed.). Matas ciliares: conservação e recuperação. 3.ed. São Paulo: Edusp/Fapesp, 2004. p.235-248.

SANTARELLI, E.G. Produção de mudas de espécies nativas. In: RODRIGUES, R.R.; LEITÃO FILHO, H.F. (Ed.). Matas ciliares: conservação e recuperação. 3.ed. São Paulo: Edusp/ Fapesp, 2004. p.313-318.

SÃO PAULO. Resolução SMA-58, de 29 de dezembro de 2006. Fixa a orientação para o reflorestamento heterogêneo de áreas degradadas e dá providências correlatas. Diário Oficial Estado de São Paulo, Poder Executivo, São Paulo, 30 dez. 2006. Seção 1, p.107.

SILVA, C.V.; BILIA, D.A.C.; MALUF, A.M.; BARBEDO, C.J. Fracionamento e germinação de sementes de uvaia (Eugenia pyriformis Cambess. - Myrtaceae). Revista Brasileira de Botânica, v.26, p.213-221, 2003.

SWAINE, M.D.; WHITMORE, T.C. On the definition of ecological groups in tropical rain forests. Vegetatio, v.75, p.81-86, 1988.

VÁZQUES-YANES, C.; OROZCO-SEGOVIA, A. Patterns of seed longevity and germination in the tropical rainforest. Annual Review of Ecology and Systematics, v.24, p.69-87, 1993.

ZAMITH, L.R.; SCARANO, F.R. Produção de mudas de espécies das Restingas do município do Rio de Janeiro, RJ, Brasil. Acta Botanica Brasilica, v.18, p.161-176, 2004. 